\title{
ELECTRO-GRAVITATIONAL TECHNOLOGY VIA CHRONON FIELD
}

\author{
Eytan H. Suchard - Applied Neural Biometrics \\ Email: eytansuchard@gmail.com \\ Pone: +972-774241773 \\ Cellphone: +97253691020
}

\section{ABSTRACT}

Aim: To develop a model of matter that will account for electro-gravity.

Note: Please note that this paper is based on the published one in

http://www.sciencedomain.org "Physical Science International Journal" to whom the author owes acknowledgement of the dramatic improvement due to a high standard and professional peer review process. The differences from the other published paper are three. 1. Correction of a qualitative classical limit calculation, term (1) which is of less importance. 2.

Transformation matrix B and not A after term (12). This is a small correction. 3. Constant improvement due to feedback from a colleague. The theory and the mathematical terms are the same.

Matter is characterized by force fields and in non-inertial and non-geodesic motion as a result of interactions. The measurement of how non geodesic a test particle is can be done by nongeodesic acceleration which in 4 dimensional space-time is perpendicular to the 4-velocity. In order to give a new meaning to matter by using such acceleration there is a need to reach a formalism free of specific trajectories, namely by a scalar curvature field. This can be done by an introduction of a new meaning of time that can't be realized as a coordinate. From every event, we define the limit of the maximal possible measurable proper time back to the "big bang" singularity or manifold of events from which we can say cosmic expansion had began. Yet such time is not a physical observable in the sense that it can't be locally calculated and it may exist as a limit only. The gradient of such time, however, is local and thus "physical". If more than one curve measuring such time cross the same event then the gradient which is a vector field can't be parallel to all such intersecting trajectories. That implies that the gradient as a vector field will not be parallel to itself or in other words, will manifest a curvature field. This idea leads to a new formalism of matter that replaces the conventional stress-energymomentum-tensor. The formalism will be mainly developed for classical but also for quantum physics and will result in a theory of electro-gravity. 


\section{INTRODUCTION}

Before we continue, we have to define the "big bang".

Big Bang: The Big Bang in this paper is a presumed event or manifold of events, such that looking backwards from any clock "Test Particle", at an event 'e', that measures the maximum possible time to 'e' must have started the measurement from the big bang as a limit. The Big Bang synchronizes all possible such clocks that measure the maximal time to any event. The idea of a test particle measuring time and even transferring time is not new, thanks to Sam Vaknin's dissertation from 1982 in which he introduced the Chronon field [1] in an amendment to Dirac's equation.

The author asserts that any test particle that measures the maximal proper time from near the "big bang" singularity event or manifold of events, will have to undergo non-geodesic acceleration as it interacts with material fields, i.e. will not move along geodesic curves unless in vacuum. An earlier incomplete paper of the author about this inertial motion prohibition in material fields, can be found [2]. The non-inertial motion is needed for the creation of trajectory intersections which in turn are needed for the creation of matter. That is a strong claim that will have to be explained. Consider a hollow ball of mass. Since this ball has a gravitational field, then by General Relativity, the clocks tick slower on the surface of the ball and in the ball than far from the ball. So particles measuring the upper limit of measurable time from near the "big bang" event or events, will have to come from outside of the gravitational field. The problem is in the center of the ball despite its zero gravity. If an unexpected acceleration is measured at the center, it will be a negligible effect comparing to the interactions near the atoms in the ball's surface, although it may affect quantum fields and move a negligible part of their energy to the center of the hollow ball. Up to the center, the direction of the trajectory curves of test particles, measuring the upper limit of proper time, is towards the center, so the gradient of the upper limit of measurable time will have spatial nonzero coordinates in the reference frame where the ball is at rest. But at the center, due to symmetry, such a gradient will have only non zero derivative in the Schwarzschild time direction. This is only one example of possible intersection of test particle trajectory curves and of their influence on the scalar field of time. 


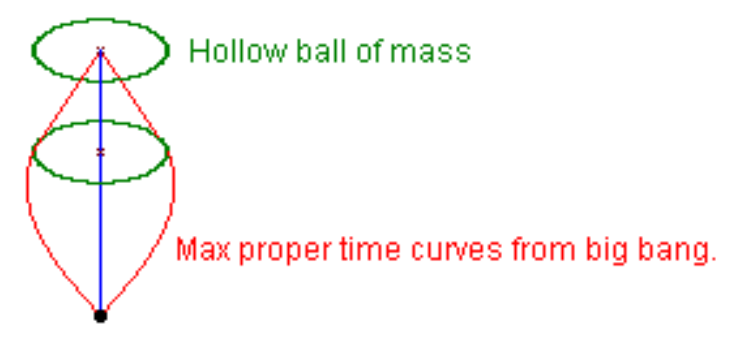

Fig. 1. The gradient of the scalar field of time is along the blue curve that is the Schwarzschild time coordinate but slightly displaced from the center, the gradient is along the red curves which result in discontinuity unless non-geodesic curvature is involved, i.e. non-inertial acceleration.

In other words, the Euler number of the gradient of the time field is not zero [3]. To avoid such singularities, the test particles must move along non geodesic curves, i.e. experience trajectory curvature and thus a mathematical formalism of such curvature will have to be developed and will have to replace matter in Einstein's field equations, as such curvature fields become a new description of matter. It is quite known that acceleration can be seen as a curvature and therefore acceleration field is another interpretation of a curvature vector perpendicular to 4-velocity [4]. An acceleration field that acts on any particle can't be expressed as a 4-vector because a 4-vector does depend on a specific trajectory and by Tzvi Scarr and Yaakov Friedman such a field is expressible by an anti-symmetric matrix $A_{\mu \nu}=-A_{v \mu}$ such that if $V_{\mu}$ is the 4-velocity such that $V_{\mu} V^{\mu}=1$ then the 4-acceleration is actually $a_{v}=A_{\mu v} V^{\mu}$

The curvature of particles that measure the upper limit of measureable time from an event back to near the "big bang" singularity event or events will be described without explicitly mentioning acceleration as this paper will present a geometric operator on the gradient of the time scalar field.

\section{THE CLASSICAL NON-RELATIVISTIC LIMIT - MASS AT REST IN A GRAVITATIONAL FIELD}

Gravity: Gravity is the phenomenon that causes all forms of energy to be inertial if and only if they freely fall, including a projectile that starts upwards. All forms of energy including light 
appear to accelerate towards the source of gravity. Gravity is seen as a phenomenon that influences the metrics of space-time.

Mass Dependent Force: $A$ mass dependent force is a presumed force that accelerates any massive object that does not propagate at the speed of light and the force is mass dependent. The mass dependent force does not change the metrics of space-time. i.e. clocks in the field will not tick slower than clocks far from the field.

An Acceleration Field / Non-Inertial Field: An acceleration Field is Mass Dependent Force that is not intrinsic to an object but rather appears as a property of space-time. Unlike gravity, it does not affect photons or any particle that propagates at the speed of light.

Motivation beyond this section:

For the pedant physicist there is no point in presenting a potential intrinsic to a massive object and a non-relativistic potential energy as the classical limit of a covariant theory. To such a reader the author will say that the purpose of this paper is to replace the conventional energy momentum tensor $T_{\mu v}$ - which is part of Einstein's field equation - by a tensor with fully geometric meaning. Recall Einstein field equations in his writing convention as $\frac{8 \pi K}{C^{4}} T_{\mu \nu}=R_{\mu v}-\frac{1}{2} R g_{\mu v}$ such that $K$ is the gravity constant, $C$, the speed of light, $R_{\mu \nu}$ the Ricci tensor, $g_{\mu \nu}$ the metric tensor, $R=g_{\mu \nu} R^{\mu v}$ the Ricci scalar. The replacement will be with a totally geometric tensor and thus will achieve a gravity equation which is geometric on both sides. To give a further clue, the author will say that $T_{\mu \nu}$ will be replaced by a tensor which is the result of a representative acceleration $\frac{a_{\lambda}}{C^{2}} \cdot \frac{a_{\lambda}}{C^{2}}$ seems as a curvature vector of a particle's trajectory with units of $1 /$ length but as such, it is an intrinsic property of the particle and not of a field. So we will have to derive our curvature vector from the gradient of a scalar field and not from the velocity of any specific particle. Since our new tensor will is purely geometric, the constant $\frac{K}{C^{4}}$ will be replaced by 1 . To be more precise, the equation will be written as $\frac{8 \pi K}{C^{4}}\left(\frac{a_{\mu} a_{v}+o t h e r_{-} t e r m s_{\mu \nu}}{K}\right)=R_{\mu \nu}-\frac{1}{2} R g_{\mu \nu}$ and in some special cases where electric charges are not involved, $Q=0$, another equation will be valid, 
$-8 \pi \frac{a_{\lambda} a^{\lambda}}{\mathrm{C}^{4}}=-R . \operatorname{In}$ any case this implies that $\frac{a^{2}}{K}=\frac{a_{\lambda} a^{\lambda}}{K}$ can be construed as energy density and hopefully the reader is not annoyed by the sloppy notation $a^{2}$.

The pedant reader is advised to skip to the next section to the one after the next, where he/she will encounter another field intrinsic to an object but as a non-relativistic limit that can't be ignored.

We continue with the classical non-relativistic limit, Using a potential field intrinsic to an object and gravitational pseudo-acceleration, the author pleads guilty as charged (hopefully without charge carriers) but nevertheless, the following will shed some light on the general intuition as to the expected relation between energy and acceleration fields although as a physical argument, it is not fully acceptable. We will now consider classical non-relativistic gravity and classical non-relativistic acceleration as qualitative limits that will hint at the relationship between non - inertial and non - geodesic acceleration fields and energy. The following will describe the pseudo-energy of the gravitational field by means of acceleration. A field of acceleration other than gravity has an important meaning, that geodesic motion in that field, i.e. matter, is prohibited. We will have to present a covariant formalism of such acceleration in more advanced sections of this paper, however, if such an acceleration is small enough then the very existence of an acceleration field is so fundamental that it redefines even the classical non-relativistic physics. Estimates will be discussed in the next section. If the classical non-relativistic and non-inertial acceleration caused by material fields is $a=\left(a_{x}, a_{y}, a_{z}\right)$ in $(x, y, z)$ coordinates then matter at rest will observe pseudo-acceleration by the gravity field $g=\left(g_{x}, g_{y}, g_{z}\right)$. Roughly we can consider 6 directions and $a=\left(a_{x}, a_{y}, a_{z}\right)$ can be parallel or perpendicular to the pseudo gravitational acceleration $g=\left(g_{x}, g_{y}, g_{z}\right)$. Restricting the discussion to parallel direction yields the resulting non-relativistic accelerations $\beta=\left(a_{x} \pm g_{x}, a_{y} \pm g_{y}, a_{z} \pm g_{z}\right)$ and $\beta \bullet \beta=a \bullet a \pm 2 a \bullet g+g \bullet g$ such that $a \bullet a=a_{x} a_{x}+a_{y} a_{y}+a_{z} a_{z}$ etc. 
Then by summation we have that the additional energy due to gravity is

$g \bullet g=g_{x} g_{x}+g_{y} g_{y}+g_{z} g_{z}$. Therefore a nice test will be to see if there is a linear relation between the integration of $g \bullet g$ and the classical negative potential energy.

We will calculate the integral of the square acceleration divided by the fourth power of the speed of light $\frac{a^{2}}{C^{4}} . K$ is the constant of gravity, $M$ mass, $r$ radii.

$\frac{1}{C^{4}} \iiint_{V=\text { Volume }} a^{2} d V=\frac{1}{C^{4}} \int_{0}^{r_{0}}\left(\frac{K\left(M \frac{r^{3}}{r_{0}^{3}}\right)}{r^{2}}\right)^{2} 4 \pi r^{2} d r=\frac{K}{C^{4}}\left(\frac{4 \pi}{5} \frac{K M^{2}}{r_{0}}\right)$

Now we calculate the negative potential energy $-E_{g}$,

$\int_{0}^{r_{0}}\left(\frac{K\left(M \frac{r^{3}}{r_{0}^{3}}\right)}{r}\right) 4 \pi r^{2} \frac{M}{4 \pi r_{0}^{3} / 3} d r=\left(\frac{3}{5} \frac{K M^{2}}{r_{0}}\right)=-E_{g}$

So from (1) and (2)

$\frac{K}{C^{4}} \frac{4 \pi}{5} \frac{K M^{2}}{r_{0}}=\frac{-\frac{4 \pi}{3} K E_{g}}{C^{4}}$

(3) qualitatively implies the following relation between energy and non-inertial acceleration where $\rho C^{2}$ is the energy density and $\rho$ is the mass density

$$
\frac{a^{2}}{C^{4}}=\frac{2 K \rho}{C^{2}}
$$

In special relativity, the square norm of a normalized by $C, 4$-velocity of a particle is constant $N^{2}=u_{i} u^{i}=1$ and also $N^{2},{ }_{k}=\left(u_{i} u^{i}\right),_{k}=\frac{d\left(u_{i} u^{i}\right)}{d x^{k}}=0$ such that $u^{i}=\frac{d x^{i}}{C d \tau}$ and the normalized by $C$, 4-acceleration is $a^{i}=\frac{d^{2} x^{i}}{C^{2} d \tau^{2}}$ which is $1 /$ length in units which is the curvature of a specific particle's trajectory. If $N^{2}$ was not the norm of a particle's velocity, we 
could think of another way to describe acceleration. More or less, that will be the subject of more advanced sections of this paper.

\section{THE CLASSICAL NON-RELATIVISTIC LIMIT - THE ELECTROSTATIC FIELD}

The following uses the standard definitions of electric and electrostatic fields.

What can we say about the density of the electrostatic field? We know it is

$$
\text { Energy_Density }=\frac{\varepsilon_{0}}{2} E^{2}
$$

such that $\varepsilon_{0}$ is the permittivity of vacuum and $E$ is the electrostatic field. Now (4) has a very deep meaning which is that acceleration of neutral charge-less test particles should appear also within an electric field,

$$
\frac{a^{2}}{C^{4}} \approx \frac{K \varepsilon_{0}}{C^{4}} E^{2} \Rightarrow \frac{a}{C^{2}} \approx \sqrt{K \varepsilon_{0}} \frac{|E|}{C^{2}}
$$

(6) implies a very weak acceleration i.e. mass dependent force on small enough charge-less neutral test particles, about $2.43 \mathrm{~cm} / \mathrm{sec}^{2}$ in a field of 1000000 volts over 1 millimeters distance. See Timir Data et. al. work as an elegant way to focus field lines by metal cone and plane and to observe the effect [5], however, in this paper we shall see that the amount of charges has to be small, otherwise the force becomes opposite in direction due to gravity and the non-geodesic acceleration field is masked by the other effect. This acceleration exposes non-inertial, non-gravitational acceleration of particles that can measure proper time. On its own it is not an interesting acceleration but it can explain the electric interaction as repulsive when the integration of the square acceleration increases and attractive when this integration is reduced. The author believes the acceleration of charge-less particles in an electric field is from positive to negative.

In "Electro-gravitational engine and Dark Matter" it will be shown that there is an electrogravitational effect opposite in direction to the acceleration of an uncharged particle in an electro-static field. There is at least informal evidence that the elecro-gravitational effect shows thrust of the entire dipole towards the positive direction [6] and the author does not imply asymmetrical capacitors of $1-0.1$ Pico-Farad with 45000 Volts. Such capacitors according to the calculations in the section "Electro-gravitational engine and Dark Matter" in this paper, can't manifest any measurable effect. 
Here is a testimony of Hector Luis Serrano in reply to Peter Liddicoat: "Actually by the generally accepted definition of what constitutes high vacuum 10^-6 Torr is about in the middle. This pressure is about equal to low Earth orbit. More importantly at this pressure the 'Mean Free Path' of the molecules in the chamber is far too great to support Corona/lon wind effects. We've tested from atmosphere to $10^{\wedge}-7$ Torr with no change in performance either. However, I'm glad the results have you thinking. It looks simple, but trust me it's not".

\section{THE NON-GEODESIC ACCELERATION FIELD}

It is required to achieve a curvature field without resorting to Tzvi Scarr and Yaakov Friedman representation [4] that is required for a general acceleration field. $A_{\mu v}=-A_{v \mu}$ such that if $V_{\mu}$ is the 4-velocity such that $V_{\mu} V^{\mu}=1$ then the 4-acceleration is actually $a_{v}=A_{\mu \nu} V^{\mu}$. In special relativity $V^{\mu}=\frac{\left(1, v_{x} / c, v_{y} / c, v_{z} / c\right)}{\sqrt{1-v^{2} / c^{2}}}$ such that $x, y, z$ are the well known three dimensional Cartesian coordinates, $v_{x}, v_{y}, v_{z}$ are three dimensional speed coordinates, $c$ the speed of light. The first coordinate is $1 / \sqrt{1-v^{2} / c^{2}}$ is the speed along the time axis. As we saw in the introduction, geodesic intersection by particles that measure the upper limit of time from near the "big bang" singularity event or manifold of events, causes discontinuity of the gradient $P,{ }_{\mu}=P_{\mu}=\frac{d P}{d x^{\mu}}$. However such conflicts can be avoided, if geodesic motion is prohibited in material fields. In classical terms that means an acceleration field $A_{\mu \nu}=-A_{v \mu}$ should emerge.

The following is simply an exercise in differential geometry. Considering a scalar field and $P$ its gradient $P_{\mu}=\frac{d P}{d x^{\mu}}$ in covariant writing, such that $d x^{\mu}$ are the coordinates, find the second power of the curvature of the field of curves generated by $P_{\mu}=\frac{d P}{d x^{\mu}}$. It is a problem in differential geometry that can be left for the reader as an exercise. However, if the reader 
wants to get the answer without too much effort along with some physical interpretations, he/she should read the following.

The idea is to use a scalar field of time - that represents the maximum possible time measured by test particles - back to near big bang singularity or to a manifold of events from which we can say the cosmic expansion had began - and from this non -physical observable, to generate observable local measurements.

The square curvature of a conserving vector field is defined by an arc length parameterization $t$ along the curves it forms.

Caution: This $t$ may not be the time measured by any physical particle because the scalar field from which the vector field is derived may be the result of an intersection of multiple trajectories. However, a particle follows the gradient curves will indeed measure $t$ even if its trajectory is not geodesic.

Let our time field be denoted by and let $P_{\mu}$ denote the derivative by coordinates $P$

$P_{\mu}=\frac{d P}{d x^{\mu}}$ or in Einstein convention $P,{ }_{\mu}=P_{\mu}$. Let $t$ be the arc length measured along the curves formed by the vector field $P_{\mu}$ which may not be always geodesic due to intersections as seen in Fig 1. By differential geometry, we know that the second power of curvature along these curves is simply

$C u r v^{2} \equiv \frac{d}{d t}\left(\frac{P_{\lambda}}{\sqrt{P^{k} P_{k}}}\right) \frac{d}{d t}\left(\frac{P_{\mu}}{\sqrt{P^{k} P_{k}}}\right) g^{\lambda \mu}$

such that $g^{\lambda \mu}$ is the metric tensor. For convenience we will write $N o r m \equiv \sqrt{P^{k} P_{k}}$ and $\dot{P}_{\lambda} \equiv \frac{d}{d t} P_{\lambda}$. For the arc length parameter $t$. Here it is the main trick, Norm may not be constant because $P_{\lambda}$ is NOT the 4-velocity of a specific particle due to intersections of more than one possible particle curve.

Let $W_{\lambda}$ denote:

$$
W_{\lambda}=\frac{d}{d t}\left(\frac{P_{\lambda}}{\sqrt{P^{k} P_{k}}}\right)=\frac{\dot{P}_{\lambda}}{N o r m}-\frac{P_{\lambda}}{N_{\text {Norm }}^{3}} P_{k} \dot{P}_{v} g^{k v}
$$


Obviously

$W_{\lambda} P_{k} g^{\lambda k}=\frac{\dot{P}_{\lambda} P_{k} g^{\lambda k}}{\text { Norm }}-\frac{P_{\lambda} P_{s} g^{\lambda s}}{\text { Norm }^{3}} P_{k} \dot{P}_{v} g^{k v}=\frac{\dot{P}_{\lambda} P_{k} g^{\lambda k}}{\text { Norm }}-\frac{P_{k} \dot{P}_{v} g^{k v}}{\text { Norm }}=0$

Thus

$\operatorname{Curv}^{2}=W_{\lambda} W^{\lambda}=\frac{\dot{P}_{\lambda} \dot{P}_{v} g^{\lambda v}}{\text { Norm }^{2}}-\frac{P_{\lambda} \dot{P}_{s} g^{\lambda s}}{N_{\text {orm }}{ }^{4}} P_{k} \dot{P}_{v} g^{k v}=\frac{\dot{P}_{\lambda} \dot{P}^{\lambda}}{\text { Norm }^{2}}-\left(\frac{P_{\lambda} \dot{P}^{\lambda}}{N_{o r m}^{2}}\right)^{2}$

Following the curves formed by $P_{\lambda}=P,_{\lambda}=\frac{d P}{d x^{\lambda}}$, The term $\frac{d x^{r}}{d t}=\frac{P_{\lambda}}{\text { Norm }}$ is the derivative of the normalized curve or normalized "velocity", using the upper Christoffel symbols, $P_{\lambda} ;_{r} \equiv \frac{d}{d x^{r}} P_{\lambda}-P_{s} \Gamma_{\lambda r}^{s}$

Caution: Using normalized velocity, here has a differential geometry meaning but not a physical meaning because a physical particle will not necessarily follow the lines which are generated by the curves parallel to the gradient $P_{\lambda}$ unless in vacuum. $P_{\lambda}$ may result from an intersection of curves along which particles move but may not be parallel to any one of such curves intersecting with an event !!!

$\frac{d}{d t} P_{\lambda}=\left(\frac{d}{d x^{r}} P_{\lambda}-P_{s} \Gamma_{\lambda r}^{s}\right) \frac{d x^{r}}{d t}=\left(P_{\lambda} ; r\right) \frac{P^{r}}{\text { Norm }}$ such that $x^{r}$ denotes the local coordinates.

If $P_{\lambda}$ is a conserving field then $P_{\lambda} ;_{r}=P_{r} ;_{\lambda}$ and thus $P_{\lambda},{ }_{r} P^{r}=\frac{1}{2}$ Norm $^{2},{ }_{\lambda}$ and

$$
\begin{aligned}
& \operatorname{Curv}^{2}=\frac{\dot{P}_{\lambda} \dot{P}^{\lambda}}{\operatorname{Norm}^{2}}-\left(\frac{P_{\lambda} \dot{P}^{\lambda}}{\operatorname{Norm}^{2}}\right)^{2}= \\
& \frac{1}{4}\left(\frac{\operatorname{Norm}^{2}{ }_{\lambda} \operatorname{Norm}^{2},{ }_{k} g^{\lambda k}}{\operatorname{Norm}^{4}}-\left(\frac{\text { Norm }^{2},{ }_{s} P_{r} g^{s r}}{\operatorname{Norm}^{3}}\right)^{2}\right)
\end{aligned}
$$

We define the Curvature Vector

$$
U_{m}=\frac{\left(\mathrm{P}^{\lambda} \mathrm{P}_{\lambda}\right),_{\mathrm{m}}}{\mathrm{P}^{\mathrm{i}} \mathrm{P}_{\mathrm{i}}}-\frac{\left(\mathrm{P}^{\lambda} \mathrm{P}_{\lambda}\right),{ }_{\mu} \mathrm{P}^{\mu}}{\left(\mathrm{P}^{\mathrm{i}} \mathrm{P}_{\mathrm{i}}\right)^{2}} P_{m}=\frac{\text { Norm }^{2},{ }_{\mathrm{m}}}{\text { Norm }^{2}}-\frac{\text { Norm }^{2},{ }_{\mu} \mathrm{P}^{\mu}}{\text { Norm }^{4}} P_{m}
$$

which from [4] and simple calculations, should have the meaning $\frac{1}{2} U_{m}=\frac{a_{\mu}}{C^{2}} B^{\mu}{ }_{m}$ such that $a_{m}$ denotes a 4-acceleration field that will accelerates every particle that can measure proper time and $C$ is the speed of light and $B^{\mu}{ }_{m}$ is a rotation matrix, i.e. 
$B^{\mu}{ }_{m} V^{m} B^{\lambda}{ }_{i} V^{i} g_{\mu \lambda}=V_{k} V^{k}, V_{m}$ is a vector and $g_{\mu \lambda}$ is the General Relativity metric tensor.

The curvature itself does not depend on any specific acceleration since it is a scalar field.

$\operatorname{Curv}^{2}=\frac{1}{4} U_{m} U^{m}$

Obviously $U_{\mu} P^{\mu}=0$ and therefore like 4 -acceleration that is perpendicular to 4-velocity $U_{\alpha}$ is perpendicular to $P_{\alpha}$. In its complex form (12) becomes

$\mathrm{Z}=N^{2}=\frac{\mathrm{P}_{\mu} \mathrm{P}^{* \mu}+\mathrm{P}^{*}{ }_{\mu} \mathrm{P}^{\mu}}{2}$ and $\hat{U}_{\lambda}=\frac{Z_{\lambda}}{Z}-\frac{Z_{k} P^{* k} P_{\lambda}}{Z^{2}}$

and by using $\frac{1}{2}\left(\hat{U}_{k} \hat{U}^{* k}+\hat{U}^{*}{ }_{k} \hat{U}^{k}\right)$

$\operatorname{Curv}^{2}=\frac{1}{4}\left(\frac{1}{2}\left(\hat{U}_{k} \hat{U}^{* k}+\hat{U}_{k}{ }_{k} \hat{U}^{k}\right)\right)$

Obviously $U_{\mu} P^{* \mu}=0$.

Possible sources for an acceleration field: An acceleration field can be represented by the Tzvi Scarr and Yaakov Friedman [4] matrix as $A^{\mu}{ }_{\nu} \frac{P^{v}}{\sqrt{P_{k} P^{k}}}=U^{\mu}$ such that $A^{\mu}{ }_{\nu}=-A^{v}{ }_{\mu}$ and $A^{\mu}{ }_{\nu}$ is a representation matrix of rotation and scaling.

The source of this field is possibly the Sam Vaknin's Chronon field [1]. In every coordinate system there exists a representation of acceleration that requires 3 parameters, $a, b, c$ that can also be complex, and takes the "Quaternion" form,

$A^{\mu}{ }_{\nu}=a A 1^{\mu}{ }_{\nu}+b A 2^{\mu}{ }_{\nu}+c A 3^{\mu}{ }_{\nu}$ and such that $A^{\mu}{ }_{\nu} \frac{P^{v}}{\sqrt{P_{k} P^{k}}}=U^{\mu}$ and

$A 1 \cdot A 2=A 3, A 1 \cdot A 3=-A 2, A 2 \cdot A 3=A 1, A 2 \cdot A 1=-A 3, A 3 \cdot A 1=A 2, A 3 \cdot A 2=-A 1$, $A 1 \cdot A 1=-I, A 2 \cdot A 2=-I, A 3 \cdot A 3=-I$ such that $I$ is the identity matrix. See "Appendix - Acceleration field representation". This implies a simpler physics and it is possible that Dirac's equation [7] and spinors are an algebraic language that was required because concurrent physics theories do not have a complete analytic theory of space-time. The need for algebraic abstraction may not be related to physical reality but rather to the way we perceive it. This possibility justifies further extensive research and should not be dismissed. 


\section{Vaknin's Theory:}

The coming definitions will reflect Dr. Sam Vaknin's view, that even photons by entanglement of wave functions have rest mass. Not that a photon as observed on its own has rest mass. That is incorrect. Matter by Vaknin's theory [1] is a result of interaction of a field of time that quite reminds of Quarks in which summation results in positive propagation. This paper sees Dr. Sam Vaknin's theory as a starting point.

Dr. Sam Vaknin's possible description of time: "Time as a wave function with observermediated collapse. Entanglement of all Chronons at the exact "monent" of the Big Bang. A relativistic QFT with Chronons as Field Quanta (excited states.) The integration is achieved via the quantum superpositions".

Energy Conservation: In any physical system and its interaction, the sum of kinetic (visible) and latent (dark) energy is constant, gain of energy is maximal and loss of energy is minimal. See E. E. Escultura [8].

Information: Information is a mathematical representation of the state of a physical system with as few labels as possible. Labels can be numbers or any other mathematical object.

Energy Density: We define $-\frac{C^{4}}{K} C u r v^{2}$, such that $C$ is the speed of light and $K$ is the gravity constant, as the Energy Density of space-time. If this value is defined by (13) then $P=\tau$ is the upper limit of measurable time from an event back to near big bang event or manifold of events and therefore (13) is intrinsic to the space-time manifold because it is dictated by the equations of gravity and adds no information that is not included in the manifold and in the equations. As we shall see, if we choose to write $P=\tau \psi$ such that $\psi$ is a complex scalar field then if $\psi$ is a function of $\tau$ only then (15) is reduced to (13) as if $P=\tau$. Consider the set of events for which $\tau$ is constant. Since $\tau$ is not a coordinate, we can't expect that set to be a sub-manifold but a unification of such 3 dimensional geometric objects $\Omega^{3}\left(\tau_{0}\right)=\Omega^{3}\left(\tau=\tau_{0}\right)$.

We consider $\tau$ as a Morse function on the space-time $M$ manifold. That is that $M \rightarrow \tau$ is locally smooth and the differential of this map is of rank 1. In such a case the Morse - Sard theorem states that the Lebesgue measure of the Critical Points of $M$ is zero [9]. 
Energy: The following is equivalent to rest mass energy. The integration of $-\int_{\Omega^{3}\left(\tau_{0}\right)} \frac{C^{4}}{K} \operatorname{Curv}^{2} d \Omega^{3}\left(\tau_{0}\right)$ is defined as the Energy of the scalar $\tau=\tau_{0}$. This value is locally conserved for small neighborhoods in $\Omega^{3}\left(\tau_{0}\right)$ if $U^{m} ;_{m}=0$ as any local integration of the squared norm of a vector field is conserved of its divergence is zero. So if there is a possibility of $U^{m} ;_{m} \neq 0$ local conservation of the term Energy does not hold. Photons too, by entanglement and superposition have rest mass but not as an isolated electro-magnetic wave.

\section{INVARIANCE UNDER DIFFERENT FUNCTIONS OF $P$}

$U_{m}=\frac{N^{2},_{m}}{N^{2}}-\frac{N^{2},{ }_{\mu} P^{\mu}}{N^{4}} P_{m}$ s.t. $N^{2} \equiv P^{i} P_{i}$ (also found as $Z$ in this paper) we can sloppily omit the comma for the sake of brevity the same way we write $\mathrm{P}_{\mathrm{i}}$ instead of $\mathrm{P},{ }_{\mathrm{i}}$ for $\frac{d P}{d x^{i}}$ and write $U_{m}=\frac{N^{2}{ }_{m}}{N^{2}}-\frac{N^{2}{ }_{\mu} P^{\mu}}{N^{4}} P_{m}$. Suppose that we replace $P$ by $f(P)$ such that $f$ is positive increasing, then $f(P)_{i} \equiv \frac{d f(P)}{d x^{i}}=\frac{d f(p)}{d p} \frac{d P}{d x^{i}}=f_{p}(P) P_{i}$. Let $N^{2} \equiv \mathrm{P}^{\lambda} \mathrm{P}_{\lambda}$ then $\hat{N}^{2} \equiv f(P)_{h} f(P)^{2}=N^{2} f_{p}(P)^{2}$ and $\frac{\hat{N}^{2}{ }_{k}}{\hat{N}^{2}}=\frac{N^{2}{ }_{k}}{N^{2}}+\frac{2 f_{p p}(p)}{f_{p}(p)} p_{k}$ but also

$\hat{U}_{k}=\frac{\hat{N}^{2}{ }_{k}}{\hat{N}^{2}}-\frac{\hat{N}^{2}{ }_{s}}{\hat{N}^{2}} \frac{f_{p}(p) p^{s} f_{p}(p) p_{k}}{\hat{N}^{2}}=$

$\frac{N^{2}{ }_{k}}{N^{2}}+\frac{2 f_{p p}(p)}{f_{p}(p)} p_{k}-\left(\frac{N^{2}{ }_{s}}{N^{2}}+\frac{2 f_{p p}(p)}{f_{p}(p)} p_{s}\right) \frac{f_{p}(p) p^{s} f_{p}(p) p_{k}}{N^{2} f_{p}(p)^{2}}=$

$\frac{N^{2}{ }_{k}}{N^{2}}-\frac{N^{2}{ }_{\mu} P^{\mu}}{N^{4}} P_{k}=U_{k}$

Consider quantum coupling between the wave function $\psi$ of a particle and the time field $P=\tau, P P^{*}=\tau^{2} \psi \psi^{*}$ is as follows Where does this coupling $P=\tau \psi$ come from ? It is has some common sense if we say that the sum of wave functions that intersect/coincide with an 
event, influence the time measurement from near the "big bang" singularity event or manifold of events to that specific event.

$$
\hat{U}_{k} \equiv\left(\frac{\hat{N}^{2}{ }_{\mathrm{k}}}{\hat{N}^{2}}-\frac{\hat{N}^{2}{ }_{\mathrm{j}}(\tau \psi)^{* \mathrm{j}}}{\left(\hat{N}^{2}\right)^{2}}(\tau \psi)_{k}\right)
$$

Index $k$, means derivative by coordinate $x^{k}, \hat{N}^{2}=(\tau \psi)_{k}\left(\tau \psi^{*}\right)^{k}, N^{2}=\tau_{k} \tau^{k}$.

As a special case, we replace $\psi$ by a wave function that depends on $\tau$ only $\psi=e^{\frac{-i E \tau}{\hbar}}$ s.t. $\quad \mathrm{i}=\sqrt{-1}$

$E$ is the energy of a coupled particle, $\hbar$ is the Barred Planck constant, so we have

$$
\begin{aligned}
& (\tau \psi)_{k}=\tau_{k} \psi+\tau \psi_{k}=\tau_{k} \psi\left(1-\frac{i \tau E}{\hbar}\right) \\
& \hat{N}^{2}=\tau_{k} \tau^{k}\left(1+\frac{\tau^{2} E^{2}}{\hbar^{2}}\right)=N^{2}\left(1+\frac{\tau^{2} E^{2}}{\hbar^{2}}\right)
\end{aligned}
$$

and

$$
\frac{\hat{N}_{s}{ }_{s}}{\hat{N}^{2}}=\frac{N_{s}^{2}}{N^{2}} \frac{\left(1+\frac{\tau^{2} E^{2}}{\hbar^{2}}\right)}{\left(1+\frac{\tau^{2} E^{2}}{\hbar^{2}}\right)}+\frac{2 \tau \tau_{s} E^{2} N^{2} / \hbar^{2}}{\left(1+\frac{\tau^{2} E^{2}}{\hbar^{2}}\right) N^{2}}=\frac{N^{2}{ }_{s}}{N^{2}}+\frac{2 \tau \tau_{s} E^{2}}{\left(\hbar^{2}+\tau^{2} E^{2}\right)}
$$

Now we want to calculate $\frac{\hat{N}_{\mathrm{j}}^{2}(\tau \psi)^{* \mathrm{j}}}{\left(\hat{N}^{2}\right)^{2}}(\tau \psi)_{k}$ so we have

$$
\begin{aligned}
& \frac{\hat{N}^{2}{ }_{\mathrm{j}}(\tau \psi)^{* \mathrm{j}}}{\left(\hat{N}^{2}\right)^{2}}(\tau \psi)_{k}= \\
& \left.\left(\frac{N^{2}{ }_{j}}{N^{2}}+\frac{2 \tau \tau_{j} E^{2}}{\left(\hbar^{2}+\tau^{2} E^{2}\right)}\right) \frac{\left(\tau^{j} \psi^{*}\left(1+\frac{i \tau E}{\hbar}\right)\right)}{\left(1+\frac{\tau^{2} E^{2}}{\hbar^{2}}\right) N^{2}} \tau_{k} \psi\left(1-\frac{i \tau E}{\hbar}\right)\right)= \\
& \left(\frac{N^{2}{ }_{j}}{N^{2}}+\frac{2 \tau \tau_{j} E^{2}}{\left(\hbar^{2}+\tau^{2} E^{2}\right)}\right) \frac{\tau^{j}}{N^{2}} \tau_{k}=\frac{N^{2}{ }_{j} \tau^{j} \tau_{k}}{\left(N^{2}\right)^{2}}+\frac{2 \tau \tau_{k} E^{2}}{\left(\hbar^{2}+\tau^{2} E^{2}\right)}
\end{aligned}
$$

From (17), (21) and (22) we have the result 


$$
\begin{aligned}
& \hat{U}_{k} \equiv\left(\frac{\hat{N}^{2}{ }_{\mathrm{k}}}{\hat{N}^{2}}-\frac{\hat{N}^{2}{ }_{\mathrm{j}}(\tau \psi)^{* \mathrm{j}}}{\left(\hat{N}^{2}\right)^{2}}(\tau \psi)_{k}\right)= \\
& \left(\left(\frac{N^{2}{ }_{k}}{N^{2}}+\frac{2 \tau \tau_{k} E^{2}}{\left(\hbar^{2}+\tau^{2} E^{2}\right)}\right)-\left(\frac{N^{2}{ }_{j} \tau^{j} \tau_{k}}{\left(N^{2}\right)^{2}}+\frac{2 \tau \tau_{k} E^{2}}{\left(\hbar^{2}+\tau^{2} E^{2}\right)}\right)\right)= \\
& \left(\frac{N^{2}{ }_{k}}{N^{2}}-\frac{N^{2}{ }_{j} \tau^{j} \tau_{k}}{\left(N^{2}\right)^{2}}\right)=\left(\frac{N^{2} k}{N^{2}}-\frac{N^{2}{ }_{j} P^{j} P_{k}}{\left(N^{2}\right)^{2}}\right)=U_{k}
\end{aligned}
$$

\section{GENERAL RELATIVITY FOR THE DETERMINISTIC LIMIT}

By General Relativity, We have to add the Hilbert-Einstein action to the negative sign of the square curvature of the gradient of the time field. Negative means that the curvature operator is mostly negative.

$$
\begin{aligned}
& \mathrm{Z}=N^{2}=\mathrm{P}_{\mu} \mathrm{P}^{\mu} \text { and } U_{\lambda}=\frac{Z_{\lambda}}{Z}-\frac{Z_{k} P^{k} P_{\lambda}}{Z^{2}} \text { and } \mathrm{L}=\frac{1}{4} \mathrm{U}^{\mathrm{k}} \mathrm{U}_{\mathrm{k}} \\
& R=\text { Riccicurvature. } \\
& \text { Min Action }=\operatorname{Min} \int_{\Omega}\left(\frac{1}{2} R-8 \pi L\right) \cdot \sqrt{-g} d \Omega
\end{aligned}
$$

A reader that still insists on asking on where does $\tau \psi$ come from, can understand that $L$ can be developed also for $\tau \psi$ and remain invariant if $\psi$ is only a smooth function of $\tau$. If

$$
\begin{aligned}
& P=\tau \psi \text { then } L=\frac{1}{8}\left(\mathrm{U}^{\mathrm{k}} \mathrm{U}^{*}{ }_{\mathrm{k}}+\mathrm{U}^{* \mathrm{k}} \mathrm{U}_{\mathrm{k}}\right) \text { and an integration constraint can be } \\
& \int_{\Omega^{3}(\tau)} \psi \psi * \sqrt{-g} d \Omega^{3}(\tau)=1
\end{aligned}
$$

Caution: $\Omega^{3}(\tau)$ is not a sub-manifold because $\tau$ is not a local coordinate and thus the local submersion theorem [10], [11] does not hold. However, $\Omega^{3}(\tau)$ is necessarily a countable unification of three dimensional sub-manifolds - Almost Everywhere - on which $\tau$ is stationary due to dimensionality considerations.

$R$ is the Ricci curvature [12], [13] and $\sqrt{-g}$ is the determinant of the metric tensor used for the 4-volume element as in tensor densities [14].

Note: If instead of $P=\tau \psi$ we choose $P=\sqrt{\tau} \psi$ then $U_{k}$ in (23) is the same if $\psi$ depends only on $\tau$. Moreover 
Instead of the constraint in (24.1) the following $\int_{\Omega^{4}(\tau)} \psi \psi * \sqrt{-g} d \Omega^{4}=1$ leads to a theory in which $\psi$ represents an event and $\int_{\Omega^{4}(\tau)} P P^{*} \sqrt{-g} d \Omega^{4}=\tau_{\text {Event }}$ represents the time of an event, i.e. see "Appendix - Event Theory". Now we return to (24). By Euler Lagrange,

$$
\begin{aligned}
& L=\frac{\left(P^{\lambda} Z_{\lambda}\right)^{2}}{Z^{3}} \text { s.t. } Z=P_{\mu} P^{\mu} \\
& \frac{\partial(L \sqrt{-g})}{\partial g^{\mu v}}-\frac{d}{d x^{m}} \frac{\partial(L \sqrt{-g})}{\partial\left(g^{\mu v},_{m}\right)}= \\
& \left(\begin{array}{l}
-2\left(\frac{\left(P^{\lambda} P_{\lambda}\right),_{s} P^{s}}{Z^{3}} P_{\mu} P_{v} P^{m}\right) ;_{m}+2 \frac{\left(P^{\lambda} P_{\lambda}\right),_{s} P^{s}}{Z^{3}}\left(\Gamma_{\mu}{ }_{m}{ }_{m} P_{i} P_{v} P^{m}+\Gamma_{v}{ }_{m} P_{\mu} P_{i} P^{m}\right)+ \\
+2\left(\frac{\left(P^{\lambda} P_{\lambda}\right),_{s} P^{s}}{Z^{3}} P_{\mu} P_{v}\right) \dot{s}_{m} P^{m}-2 \frac{\left(P^{\lambda} P_{\lambda}\right),_{s} P^{s}}{Z^{3}}\left(\Gamma_{\mu}{ }_{m}{ }_{m} P_{i} P_{v} P^{m}+\Gamma_{v}{ }_{m}{ }_{m} P_{\mu} P_{i} P^{m}\right)+ \\
+2\left(\frac{\left(P^{\lambda} P_{\lambda}\right){ }_{s} P^{s}}{Z^{3}}\right) Z_{\mu} P_{v}-3\left(\frac{\left(\left(P^{\lambda} P_{\lambda}\right),_{s} P^{s}\right)^{2}}{Z^{4}}\right) P_{\mu} P_{v}-\frac{1}{2} \frac{\left(P^{\lambda} Z_{\lambda}\right)^{2}}{Z^{3}} g_{\mu v}
\end{array}\right) \\
& =\left(\begin{array}{l}
-2\left(\frac{\left(P^{\lambda} P_{\lambda}\right){ }_{m} P^{m}}{Z^{3}} P^{k}\right) ;_{k} P_{\mu} P_{v}-2 \frac{\left(P^{\lambda} Z_{\lambda}\right)^{2}}{Z^{3}} \frac{P_{\mu} P_{v}}{Z}+2\left(\frac{\left(P^{\lambda} P_{\lambda}\right){ }_{s} P^{s}}{Z^{3}}\right) Z_{\mu} P_{v}+ \\
-\frac{1}{2} \frac{\left(P^{\lambda} Z_{\lambda}\right)^{2}}{Z^{3}} g_{\mu v}-\frac{\left(P^{\lambda} Z_{\lambda}\right)^{2}}{Z^{3}} \frac{P_{\mu} P_{v}}{Z}
\end{array}\right) \sqrt{-g}
\end{aligned}
$$

$$
\begin{aligned}
& L=\frac{Z^{\lambda} Z_{\lambda}}{Z^{2}} \text { s.t. } Z=P_{\mu} P^{\mu} \\
& \frac{\partial(L \sqrt{-g})}{\partial g^{\mu v}}-\frac{d}{d x^{m}} \frac{\partial(L \sqrt{-g})}{\partial g^{\mu \nu}{ }_{m}}= \\
& \left(\begin{array}{l}
\left.-2\left(\frac{Z^{m} P_{\mu} P_{v}}{Z^{2}}\right) ;_{m}+2 \frac{\left(\Gamma_{\mu}{ }_{m}{ }_{m} P_{i} P_{v} Z^{m}+\Gamma_{v}{ }_{m}{ }_{m} P_{\mu} P_{i} Z^{m}\right)}{Z^{2}}\right)+ \\
\left.+2 \frac{\left.\left(P_{\mu} P_{v}\right) \dot{m}_{m} Z^{m}\right)}{Z^{2}}-2 \frac{\left(\Gamma_{\mu}{ }_{m}{ }_{m} P_{i} P_{v} Z^{m}+\Gamma_{v}{ }_{m}{ }_{m} P_{\mu} P_{i} Z^{m}\right)}{Z^{2}}\right)+ \\
+\frac{Z_{\mu} Z_{v}}{Z^{2}}-2 \frac{Z_{s} Z^{s}}{Z^{3}} P_{\mu} P_{v}-\frac{1}{2} \frac{Z_{m} Z^{m}}{\left(P^{i} P_{i}\right)^{2}} g_{\mu v}
\end{array}\right) \sqrt{-g}= \\
& \left(-2\left(\frac{Z^{m}}{Z^{2}}\right) ;_{m} P_{\mu} P_{v}-2 \frac{Z^{\lambda} Z_{\lambda}}{Z^{2}} \frac{P_{\mu} P_{v}}{Z}-\frac{1}{2} \frac{Z_{k} Z^{k}}{Z^{2}} g_{\mu v}+\frac{Z_{\mu} Z_{v}}{Z^{2}}\right) \sqrt{-g}
\end{aligned}
$$




$$
\begin{aligned}
& Z=P_{\mu} P^{\mu} \text { and } U_{\lambda}=\frac{Z_{\lambda}}{Z}-\frac{Z_{k} P^{k} P_{\lambda}}{Z^{2}} \text { and } L=U^{\kappa} U_{k} \\
& \frac{\partial(L \sqrt{-g})}{\partial g^{\mu v}}-\frac{d}{d x^{m}} \frac{\partial(L \sqrt{-g})}{\partial g^{\mu v},_{m}}= \\
& \left(+2\left(\frac{\left(P^{\lambda} P_{\lambda}\right){ }_{m} P^{m}}{Z^{3}} P^{k}\right) ;_{k} P_{\mu} P_{v}+2 \frac{\left(P^{\lambda} Z_{\lambda}\right)^{2}}{Z^{3}} \frac{P_{\mu} P_{v}}{Z}-2\left(\frac{\left(P^{\lambda} P_{\lambda}\right){ }_{s} P^{s}}{Z^{3}}\right) Z_{\mu} P_{v}+\right. \\
& +\frac{1}{2} \frac{\left(P^{\lambda} Z_{\lambda}\right)^{2}}{Z^{3}} g_{\mu v}+\frac{\left(P^{\lambda} Z_{\lambda}\right)^{2}}{Z^{3}} \frac{P_{\mu} P_{v}}{Z}+ \\
& \left(-2\left(\frac{Z^{m}}{Z^{2}}\right) ;_{m} P_{\mu} P_{v}-2 \frac{Z^{\lambda} Z_{\lambda}}{Z^{2}} \frac{P_{\mu} P_{v}}{Z}-\frac{1}{2} \frac{Z_{k} Z^{k}}{Z^{2}} g_{\mu v}+\frac{Z_{\mu} Z_{v}}{Z^{2}}\right) \\
& \left(+2\left(\left(\frac{\left(P^{\lambda} P_{\lambda}\right),_{m} P^{m}}{Z^{3}} P^{k}\right) ;_{k}-2\left(\frac{Z^{m}}{Z^{2}}\right) ;_{m}\right) P_{\mu} P_{v}+\right. \\
& +2 \frac{\left(P^{\lambda} Z_{\lambda}\right)^{2}}{Z^{3}} \frac{P_{\mu} P_{v}}{Z}-2 \frac{Z^{\lambda} Z_{\lambda}}{Z^{2}} \frac{P_{\mu} P_{v}}{Z}+ \\
& +\frac{1}{2} \frac{\left(P^{\lambda} Z_{\lambda}\right)^{2}}{Z^{3}} g_{\mu \nu}-\frac{1}{2} \frac{Z_{k} Z^{k}}{Z^{2}} g_{\mu \nu}+ \\
& \left.+\frac{Z_{\mu} Z_{v}}{Z^{2}}-2\left(\frac{\left(P^{\lambda} P_{\lambda}\right),_{s} P^{s}}{Z^{3}}\right) Z_{\mu} P_{v}+\frac{\left(P^{\lambda} Z_{\lambda}\right)^{2}}{Z^{3}} \frac{P_{\mu} P_{v}}{Z}\right) \\
& \left(+2\left(\left(\frac{\left(P^{\lambda} P_{\lambda}\right){ }_{m} P^{m}}{Z^{3}} P^{k}\right) ;_{k}-2\left(\frac{Z^{m}}{Z^{2}}\right) ;_{m}\right) P_{\mu} P_{v}+\right. \\
& \left(\begin{array}{l}
+2 \frac{\left(P^{\lambda} Z_{\lambda}\right)^{2}}{Z^{3}} \frac{P_{\mu} P_{v}}{Z}-2 \frac{Z^{\lambda} Z_{\lambda}}{Z^{2}} \frac{P_{\mu} P_{v}}{Z}+ \\
+U_{\mu} U_{v}-\frac{1}{2} U_{k} U^{k} g_{\mu v}
\end{array}\right) \cdot \sqrt{-g}= \\
& \left(U_{\mu} U_{v}-\frac{1}{2} U_{k} U^{k} g_{\mu \nu}-2 U^{k} ;_{k} \frac{P_{\mu} P_{v}}{Z}\right) \sqrt{-g}
\end{aligned}
$$

(27) 


$$
\begin{aligned}
& L=\frac{\left(Z^{s} P_{s}\right)^{2}}{Z^{3}} \text { s.t. } Z=P^{\lambda} P_{\lambda} \text { and } Z_{m}=\left(P^{\lambda} P_{\lambda}\right)_{m} \\
& \frac{\partial(L \sqrt{-g})}{\partial P_{\mu}}-\frac{d}{d x^{v}} \frac{\partial(L \sqrt{-g})}{\partial P_{\mu},_{v}}= \\
& \left(\begin{array}{l}
-4\left(\frac{\left(Z_{s} P^{s}\right)}{Z^{3}} P^{\mu} P^{v}\right) ;_{v}+4 \frac{\left(Z_{s} P^{s}\right)}{Z^{3}} \Gamma_{i}^{\mu}{ }_{v} P^{i} P^{v}+ \\
+4 \frac{\left(Z_{s} P^{s}\right)}{Z^{3}} P^{\mu}{ }_{{ }_{v}} P^{v}-4 \frac{\left(Z_{s} P^{s}\right)}{Z^{3}} \Gamma_{i}{ }_{k}{ }_{k} P^{i} P^{k}+ \\
+2 \frac{Z_{m} P^{m} Z^{\mu}}{Z^{3}}-6 \frac{\left(Z_{m} P^{m}\right)^{2}}{Z^{4}} P^{\mu}
\end{array}\right) \sqrt{-g}= \\
& \left(-4\left(\frac{\left(Z_{s} P^{s}\right) P^{v}}{Z^{3}}\right) ;_{v} P^{\mu}+2 \frac{Z_{m} P^{m} Z^{\mu}}{Z^{3}}-6 \frac{\left(Z_{m} P^{m}\right)^{2}}{Z^{4}} P^{\mu}\right) \sqrt{-g} \\
& L=\frac{Z^{s} Z_{s}}{Z^{2}} \text { s.t. } Z=P^{\lambda} P_{\lambda} \text { and } Z_{m}=\left(P^{\lambda} P_{\lambda}\right)_{m} \\
& \frac{\partial(L \sqrt{-g})}{\partial P_{\mu}}-\frac{d}{d x^{v}} \frac{\partial(L \sqrt{-g})}{\partial P_{\mu}{ }_{\nu}}= \\
& \left(\begin{array}{l}
-4\left(\frac{P^{\mu} Z^{v}}{Z^{2}}\right) ;_{v}+\frac{4}{Z^{2}} \Gamma_{i}^{\mu}{ }_{k} P^{i} Z^{k}+ \\
+\frac{4}{Z^{2}} P^{\mu} ;_{v} Z^{v}-\frac{4}{Z^{2}} \Gamma_{i}^{\mu}{ }_{k} P^{i} Z^{k}+ \\
-4 \frac{Z_{m} Z^{m}}{Z^{3}} P^{\mu} \sqrt{-g}
\end{array}\right) \sqrt{-g}= \\
& \left(-4\left(\frac{Z^{v}}{Z^{2}}\right) ;_{v}-4 \frac{Z_{m} Z^{m}}{Z^{3}}\right) P^{\mu} \sqrt{-g}
\end{aligned}
$$

From (24) and (27), 


$$
\begin{aligned}
& \mathrm{Z}=N^{2}=\mathrm{P}_{\mu} \mathrm{P}^{\mu}, U_{\lambda}=\frac{Z_{\lambda}}{Z}-\frac{Z_{k} P^{k} P_{\lambda}}{Z^{2}}, L=\frac{1}{4} U_{i} U^{i} \text { and } \mathrm{Z}=\mathrm{P}^{\mathrm{k}} \mathrm{P}_{\mathrm{k}} \\
& \frac{8 \pi}{4}\left(\begin{array}{l}
+2\left(\left(\frac{\left(\mathrm{P}^{\lambda} \mathrm{P}_{\lambda}\right),_{\mathrm{m}} P^{m}}{Z^{3}} P^{k}\right) ;_{k}-2\left(\frac{Z^{m}}{Z^{2}}\right) \dot{m}_{m}\right) P_{\mu} P_{v}+ \\
+2 \frac{\left(P^{\lambda} Z_{\lambda}\right)^{2}}{Z^{3}} \frac{P_{\mu} P_{v}}{Z}-2 \frac{Z^{\lambda} Z_{\lambda}}{Z^{2}} \frac{P_{\mu} P_{v}}{Z}+ \\
+U_{\mu} U_{v}-\frac{1}{2} U_{\mathrm{k}} U^{k} g_{\mu v}
\end{array}\right) .= \\
& \frac{8 \pi}{4}\left(U_{\mu} U_{v}-\frac{1}{2} U_{\mathrm{k}} U^{k} g_{\mu v}-2 U^{k} ;{ }_{k} \frac{P_{\mu} P_{v}}{Z}\right)=R_{\mu v}-\frac{1}{2} R g_{\mu v} \\
& \text { s.t. } R=R_{\mu v} g^{\mu v} \\
& \text { s.t. } R_{k j}=\left(\Gamma_{j k}{ }^{P}\right),{ }_{p}-\left(\Gamma_{p k}{ }^{P}\right),{ }_{j}+\Gamma_{p \mu}{ }^{P} \Gamma_{j k}{ }^{\mu}-\Gamma_{p j}{ }^{\mu} \Gamma_{k \mu}{ }^{p}
\end{aligned}
$$

$R_{\mu \nu}$ is the Ricci tensor. From (24),(28),(29) we have,

$$
\frac{d}{d x^{\mu}}\left(\frac{\partial}{\partial P_{\mu}}-\frac{d}{d x^{v}} \frac{\partial}{\partial P_{\mu},{ }_{\nu}}\right)\left(U_{k} U^{k} \sqrt{-g}\right)=W^{\mu} ;{ }_{\mu} \sqrt{-g}=0
$$

$$
W^{\mu} ;_{\mu}=\left(\begin{array}{l}
4\left(\frac{\left(Z_{s} P^{s}\right) P^{v}}{Z^{3}}\right) ;_{\nu} P^{\mu}+4 \frac{\left(Z_{m} P^{m}\right)^{2}}{Z^{4}} P^{\mu}+ \\
-4\left(\frac{Z^{v}}{Z^{2}}\right){ }_{\nu} P^{\mu}-4 \frac{Z_{\mathrm{m}} Z^{m}}{Z^{3}} \mathrm{P}^{\mu}+ \\
2 \frac{\left(Z_{m} P^{m}\right)^{2}}{Z^{4}} P^{\mu}-2 \frac{Z_{\mathrm{m}} P^{m} Z^{\mu}}{Z^{3}}
\end{array}\right) ;{ }_{\mu}=0
$$

(31) is equivalent to $W^{\mu} ;_{\mu}=\left(-4 U^{\nu} ;_{\nu} \frac{P^{\mu}}{Z}-2 \frac{\left(Z_{m} P^{m}\right)}{Z^{2}} U^{\mu}\right) ;_{\mu}=0$

A simpler solution to zero Euler Lagrange equations, is

$$
\left(\frac{\partial}{\partial P_{\mu}}-\frac{d}{d x^{v}} \frac{\partial}{\partial P_{\mu},_{v}}\right)\left(U_{k} U^{k} \sqrt{-g}\right)=0
$$

Which results in a special case, "Zero Charges" as charges are related to non-zero divergences,

$$
\left(U^{v}\right){ }_{v}=0
$$

and (30) becomes,

$$
\frac{8 \pi}{4}\left(U_{\mu} U_{v}-\frac{1}{2} U_{\mathrm{k}} U^{k} g_{\mu v}\right)=R_{\mu v}-\frac{1}{2} R g_{\mu \nu}
$$


The reader can either refer to the following calculation or skip it.

$$
\begin{aligned}
& \left(-4\left(\frac{Z^{v}}{Z^{2}}\right) ;_{v}-4 \frac{Z_{\mathrm{m}} Z^{m}}{Z^{3}}\right) \mathrm{P}^{\mu}+4\left(\frac{\left(Z_{s} P^{s}\right) P^{v}}{Z^{3}}\right) ;_{v} P^{\mu} \\
& -2 \frac{Z_{\mathrm{m}} P^{m} Z^{\mu}}{Z^{3}}+6 \frac{\left(Z_{m} P^{m}\right)^{2}}{Z^{4}} P^{\mu}= \\
& -4\left(\frac{Z^{v}}{Z^{2}}\right) ;_{v} \mathrm{P}^{\mu}-4 \frac{Z_{\mathrm{m}} Z^{m}}{Z^{3}} \mathrm{P}^{\mu}+ \\
& +4\left(\frac{\left(Z_{s} P^{s}\right) P^{v}}{Z^{3}}\right) ;_{v} P^{\mu}+4 \frac{\left(Z_{m} P^{m}\right)^{2}}{Z^{4}} P^{\mu} \\
& -2 \frac{Z_{\mathrm{m}} P^{m}}{Z^{2}}\left(\frac{Z^{\mu}}{Z}-\frac{Z_{m} P^{m} P^{\mu}}{Z^{2}}\right)= \\
& -4\left(\left(\frac{U^{k}}{Z}\right) ;_{k}+\frac{U^{k} U_{k}}{Z}\right) P^{\mu}-2 \frac{Z_{\mathrm{m}} P^{m}}{Z^{2}} U^{\mu}=0
\end{aligned}
$$

Recall that $U^{k} P_{k}=0$, multiplication by $\frac{-P_{\mu}}{4}$ and contraction yields,

$$
\begin{aligned}
& \left(\left(\frac{Z^{v}}{Z^{2}}\right) ;{ }_{v}-\left(\frac{\left(Z_{s} P^{s}\right) P^{v}}{Z^{3}}\right) ;{ }_{v}\right) Z+\frac{Z_{\mathrm{m}} Z^{m}}{Z^{2}}-\frac{\left(Z_{m} P^{m}\right)^{2}}{Z^{3}}=0 \\
& \left(\frac{Z^{v}}{Z^{2}}\right) ;{ }_{v}-\left(\frac{\left(Z_{s} P^{s}\right) P^{v}}{Z^{3}}\right){ }_{v}+\frac{1}{Z}\left(\frac{Z_{\mathrm{m}} Z^{m}}{Z^{2}}-\frac{\left(Z_{m} P^{m}\right)^{2}}{Z^{3}}\right)=0
\end{aligned}
$$

and as a result of (37) the following term from (30) vanishes,

$$
\begin{aligned}
& -2\left(U^{k}\right) ;_{k} \frac{P^{\mu} P^{v}}{Z}=-2\left(\frac{U^{k}}{Z}\right) ;_{k} P^{\mu} P^{v}-2 U^{k} U_{k} \frac{P^{\mu} P^{v}}{Z}= \\
& -2\left(\frac{\left(\mathrm{P}^{\lambda} \mathrm{P}_{\lambda}\right),_{\mathrm{m}} P^{m}}{Z^{3}} P^{k}\right) ;_{k} P^{\mu} P^{v}-2 \frac{\left(Z_{\mathrm{s}} P^{s}\right)^{2}}{Z^{3}} \frac{P^{\mu} P^{v}}{Z}+ \\
& 2\left(\frac{Z^{m}}{Z^{2}}\right) ;_{m} P^{\mu} P^{v}+2 \frac{Z^{\lambda} Z_{\lambda}}{Z^{2}} \frac{P^{\mu} P^{v}}{Z}= \\
& -2\left(\frac{\left(\mathrm{P}^{\lambda} \mathrm{P}_{\lambda}\right),_{\mathrm{m}} P^{m}}{Z^{3}} P^{k}\right) ;_{k} P^{\mu} P^{v}-2 \frac{\left(Z_{\mathrm{s}} P^{s}\right)^{2}}{Z^{3}} \frac{P^{\mu} P^{v}}{Z}+ \\
& 2\left(\frac{Z^{m}}{Z^{2}}\right) ;_{m} P^{\mu} P^{v}+2 \frac{Z^{\lambda} Z_{\lambda}}{Z^{2}} \frac{P^{\mu} P^{v}}{Z}= \\
& 2\left(\left(\frac{Z^{m}}{Z^{2}}\right) ;_{m}-\left(\frac{\left(\mathrm{P}^{\lambda} \mathrm{P}_{\lambda}\right){ }_{\mathrm{m}} P^{m}}{Z^{3}} P^{k}\right) ;_{k}+\frac{1}{Z}\left(\frac{Z^{\lambda} Z_{\lambda}}{Z^{2}}-\frac{\left(Z_{\mathrm{s}} P^{s}\right)^{2}}{Z^{3}}\right)\right) P^{\mu} P^{v}=0
\end{aligned}
$$

Which yields a simpler equation (34). Recall that $U^{v}=\frac{Z^{v}}{Z}-\frac{\left(Z_{s} P^{s}\right) P^{v}}{Z^{2}}$,

And that $\frac{Z_{v}}{Z} U^{v}=U_{v} U^{v}$ 


$$
\begin{aligned}
& \left(\frac{Z^{v}}{Z^{2}}\right) ;_{v}-\left(\frac{\left(Z_{s} P^{s}\right) P^{v}}{Z^{3}}\right) ;_{v}+\frac{1}{Z}\left(\frac{Z_{\mathrm{m}} Z^{m}}{Z^{2}}-\frac{\left(Z_{m} P^{m}\right)^{2}}{Z^{3}}\right)= \\
& \left(\frac{U^{v}}{Z}\right) ;_{v}+\frac{1}{Z}\left(U_{\mathrm{m}} U^{m}\right)=\frac{1}{Z}\left(U^{v}\right) ;_{v}-\frac{1}{Z^{2}} U^{v} Z_{v}+\frac{1}{Z}\left(U_{\mathrm{m}} U^{m}\right)= \\
& \frac{1}{Z}\left(U^{v}\right) ;_{v}=0
\end{aligned}
$$

Which proves (33).

Question to the reader: If $U_{\mu} U_{v}-\frac{1}{2} U_{\mathrm{k}} U^{k} g_{\mu \nu}-2 U^{k} ;_{k} \frac{P_{\mu} P_{v}}{Z}$ describes the electromagnetic energy momentum tensor, where is the torsion tensor $F_{\mu \nu}=-F_{\nu \mu}$ that is so basic to the electro-magnetic theory?

Answer: $U_{\mu}$ is not any electro-magnetic field. It is a property of space-time as $P=\tau$ is dictated by the equations of gravity. $U_{\mu}$, however, offers a way to describe an antisymmetric tensor which is a scaling and rotation matrix as an acceleration field via Tzvi Scarr and Yaakov Friedman representation [4], $U_{v}=A_{\mu \nu} \frac{P^{\mu}}{\sqrt{P^{\lambda} P_{\lambda}}}$ such that $A_{\mu \nu}=-A_{v \mu}$. See "Appendix - Acceleration field representation". The field is actually represented by $A_{\mu \nu}$ but it is not an electromagnetic field, rather, it is the underlying mechanism that results in what we call, the electric field. In the complex formalism either, $U{ }_{\nu}=A_{\mu \nu} \frac{P^{\mu}}{\sqrt{P^{\lambda} P_{\lambda}}}$ or $U_{v}=A_{\mu \nu} \frac{P^{* \mu}}{\sqrt{P^{\lambda} P_{\lambda}}}$. Increasing or decreasing $\frac{C^{4}}{8 K}\left(U{ }_{\nu} U^{v}+U_{v} U^{* v}\right)$ results in change of Energy density and in the phenomena we call Electro-Magnetism. $A_{\mu v}$ represents a noninertial acceleration of every particle that can measure proper time and not of photons as single particles.

Inertia Tensor: We define inertia tensor as $U_{\mu} U_{v}-\frac{1}{2} U_{\mathrm{k}} U^{k} g_{\mu v}$ this reflects what we know as energy momentum tensor. 
Electro gravity tensor: We define the electro-gravity tensor as $-2 U^{k} ;_{k} \frac{P_{\mu} P_{v}}{\sqrt{P^{k} P_{k}}}$.

As we shall see, $-2 U^{k} ;{ }_{k}$ is equivalent to electric charge density. $U^{k} ;{ }_{k} \approx-\sqrt{\frac{4 K}{\varepsilon_{0}}} \frac{\rho}{C^{2}}$

But the sign could be also plus. Charge conservation yields the following:

From, $\left(U_{\mu} U_{v}-\frac{1}{2} U_{\mathrm{k}} U^{k} g_{\mu v}-2 U^{k} ;_{k} \frac{P_{\mu} P_{v}}{Z}\right) g^{\mu v}=-U_{\mathrm{k}} U^{k}-2 U^{k} ;_{k}$ it follows

that $2 \pi \int_{\Omega}\left(-U_{\mathrm{k}} U^{k}-2 U^{k} ;{ }_{k}\right) \sqrt{-g} d \Omega=2 \pi \int_{\Omega}-U_{\mathrm{k}} U^{k} \sqrt{-g} d \Omega=\int_{\Omega}-R \sqrt{-g} d \Omega$.

Construction and Destruction: We define construction or destruction as local appearance and disappearance of non zero $-2 U^{k} ;_{k}$ neighborhoods of space-time as a function of $\tau$. This definition alludes to the well known terms of construction and destruction brackets in Quantum Mechanics.

\section{RESULTS - ELECTRO-GRAVITATIONAL ENGINE, DARK MATTER AND DARK ENERGY}

Dark Matter: Dark Matter will be defined as additional Gravity not due to the Inertia Tensor. It is meant that the cause of such gravity is not inertial mass that resists non-inertial acceleration.

Dark Energy: Dark Energy will be defined as negative Gravity not due to the Inertia Tensor. It is meant that the cause of such gravity is not inertial mass that resists non-inertial acceleration.

The following will describe a technology that can take energy from space-time apparently by Sciama Inertial Induction [15] and is closely related to Alcubierre Warp Drive [16]. Electrogravity follows from (6), (30) and (31). For several reasons we may assume the weak acceleration of uncharged particles mentioned in (6) is from positive to negative charges see also [5], consider the general relativity equation

$\frac{8 \pi}{4}\left(U_{\mu} U_{v}-\frac{1}{2} U_{\mathrm{k}} U^{k} g_{\mu v}-2 U^{k} ;_{k} \frac{P_{\mu} P_{v}}{Z}\right)=G_{\mu \nu}=R_{\mu \nu}-\frac{1}{2} R g_{\mu \nu}$ such that the Ricci tensor is $R_{k j}=\left(\Gamma_{j k}{ }^{P}\right),{ }_{p}-\left(\Gamma_{p k}{ }^{P}\right),{ }_{j}+\Gamma_{p \mu}{ }^{P} \Gamma_{j k}{ }^{\mu}-\Gamma_{p j}{ }^{\mu} \Gamma_{k \mu}{ }^{p}$ 
$G_{\mu \nu}$ is the Einstein tensor. From (4) in a weak gravitational background field,

$$
\frac{1}{2} U_{m}=\frac{1}{2}\left(\frac{\left(P^{\lambda} P_{\lambda}\right){ }_{m}}{P^{i} P_{i}}-\frac{\left(P^{\lambda} P_{\lambda}\right){ }_{\mu} P^{\mu}}{\left(P^{i} P_{i}\right)^{2}} P_{m}\right) \approx \frac{a}{C^{2}}=\sqrt{K \varepsilon_{0}} \frac{E_{m}}{C^{2}}
$$

$C$ is the speed of light, $a$ is the non-relativistic weak acceleration of an uncharged particle, $\varepsilon_{0}$ is the permittivity constant in vacuum, $K$ is the gravitational constant and $E$ is a static non-relativistic electric field in weak gravity, assuming that by correct choice of coordinates,

$$
E_{m}=\left(E_{0}=0, E_{1}, E_{2}, E_{3}\right)
$$

and also

$$
E^{k} ;_{0}=0
$$

From electro-magnetism

$$
E^{k} ;_{k}=\frac{\rho}{\varepsilon_{0}}
$$

Such that $\rho$ is the charge density

$$
\frac{1}{4}\left(U_{\mu} U_{v}-\frac{1}{2} U_{\mathrm{k}} U^{k} g_{\mu v}\right) \approx K \varepsilon_{0} \frac{1}{C^{4}}\left(E_{\mu} E_{v}-\frac{1}{2} E_{\mathrm{k}} E^{k} g_{\mu \nu}\right)
$$

And

$-\frac{1}{4} 2 U^{k} ;{ }_{k} \frac{P_{\mu} P_{v}}{Z} \approx \sqrt{K \varepsilon_{0}} \frac{E^{k} ;_{k}}{C^{2}} \frac{P_{\mu} P_{v}}{Z}$

From the electro-magnetic theory $E^{k} ;_{k}=\frac{\rho}{\varepsilon_{0}}$ such that $\rho$ is the charge density and so for $t$ Schwarzschild coordinate time,

$8 \pi \sqrt{\frac{K}{\varepsilon_{0}}} \frac{\rho}{C^{2}} \approx G_{t t}=G_{00}$

So $\frac{8 \pi K}{C^{4}} \sqrt{\frac{1}{\varepsilon_{0} K}} \rho C^{2} \approx G_{t t}=G_{00}$ such that

$\frac{\rho}{\sqrt{\varepsilon_{0} K}}$ behaves like mass density and therefore we can define an electro-gravitational virtual mass as dependent on charge $Q$ :

$$
M_{\text {Virtual }}=\frac{Q}{\sqrt{\varepsilon_{0} K}}
$$


We will calculate Virtual_Mass $=\frac{ \pm Q}{\sqrt{K \varepsilon_{0}}}$ for \pm 20 Coulombs.

$\frac{ \pm 1 \text { Coulomb }}{\sqrt{K \varepsilon_{0}}}= \pm 4.1137485256189825984285067001904 \times 10^{10} \mathrm{Kg}$

Multiplied by 20 we have

$\frac{ \pm 20 \text { Coulombs }}{\sqrt{K \varepsilon_{0}}}= \pm 8.2274970512379651968570134003808 \times 10^{11} \mathrm{Kg}$

Within 1 cubic meter the effect would be a feasible electro-gravitational field because Newton's gravitational acceleration as a rough approximation yields,

$\frac{K \cdot \text { Virtual_Mass }_{-}}{\text {radius }^{2}}=$

$K \cdot 8.2274970512379651968570134003808 \times 10^{11} \mathrm{Kg} / 1^{2}=$ $54.908998920433981649392210311998 \frac{\text { Meter }}{\text { Second }^{2}}$

a little less than $5 \mathrm{~g}$. The problem is the capacitance of parallel plates, Cap $=\frac{\varepsilon_{0} A}{d}$ and

Cap $\cdot$ Volt $=\mathrm{Q}$, such that Cap denotes Capacitance, $A$ is the area, $d$ is the distance, $\mathrm{V}$ is the voltage and $\mathrm{Q}$ is the charge. The solution to that problem is to use multiple parallel capacitors stacked together one on top of the other such that the material between two adjacent capacitors will have much higher permittivity than the gap between each capacitor's boards and such that all capacitors will be wired in parallel. The result is a cumulative effect of little electro-gravitational warps. This model was developed with the help of Ran Timar, Elad Dayan and Benny Versano who are electrical engineers. 


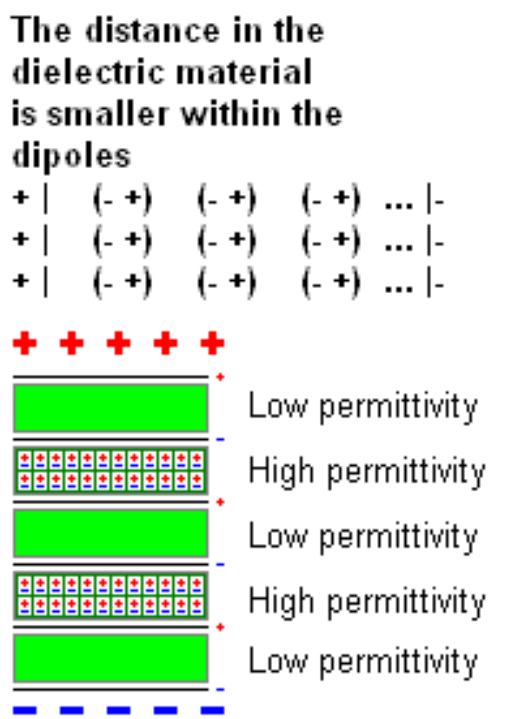

The distance in the dielectric material is smaller between different dipoles



Fig. 2. Suchard - Dayan - Timar - Versano model, the effect in the wrong direction from plus below to minus above is attenuated by dielectric slabs.

The idea: The charges in the dielectric molecules are either closer than between molecules or closer between different molecules. Best results not necessarily result from the highest possible dielectric constant. The goal is that electro-gravity will be stronger within the aligned molecules as gravity depends on the square inverse of distance. The polarity caused by the conducting boards nearly cancels out and a net effect is expected.

The calculations rule out any measurable vacuum thrust of Pico-Farad or less, asymmetrical capacitors even with 50000 volts supply, simply because the net effect depends on the total amount of separated charges which are far from sufficient in standard Biefeld Brown capacitors [17].

Use of plasma: Another idea is to use ionized plasma. Let us see what we can do with one gram of ionized hydrogen. The number of atoms by Avogadro's number is $n=6.02214129 \times 10^{23}$. The charge of the electron is $\mathrm{e}=1.602176565 \times 10^{-19}$ Coloumbsso $Q= \pm 9.64853364595686885 \times 10^{4}$ Coloumbs $K=6.67384 \times 10^{-11} \mathrm{~m}^{3} \mathrm{~kg}^{-1} \mathrm{sec}^{-1}$ and $\varepsilon_{0}=8.8541878176 . . \times 10^{-12} \mathrm{~F} / \mathrm{m}$ so $(10.23)$ reaches a virtual mass of Virtual_Mass $\approx \pm 3.9691641060440215872505202441024 \times 10^{15} \mathrm{Kg}$. That is far less than the mass of the Earth $M_{\text {Earth }}=5.97219 \times 10^{24} \mathrm{Kg}$ but the distance between two clouds 
of positive and negative ionized hydrogen can be much less than the average Earth radius and therefore a field that overcomes the Earth gravitational field is feasible.

Dark Matter and Dark Energy follow immediately from positively ionized gas in the galaxy and negatively ionized gas outside or on the outskirts.

We now consider the classical non-covariant limit of the summation of two effects, the noninertial acceleration and electro-gravity. Let $Q$ be the charge of a ball at radius $r$ then the observed acceleration of an uncharged particle $a$ without any induced diploes is $a \approx \frac{-K Q}{r^{2} \sqrt{K \varepsilon_{0}}}+\sqrt{K \varepsilon_{0}} \frac{Q}{4 \pi \varepsilon_{0} r^{2}}=\sqrt{\frac{K}{\varepsilon_{0}}} \frac{Q}{r^{2}}\left(\frac{1}{4 \pi}-1\right)$.

\section{CONCLUSION}

An upper limit on measurable time from each event backwards to the "big bang" singularity or manifold of events may exist only as a limit and is not a practical physical observable in the usual sense. Since more than one curve on which such time can be virtually measured, intersects the same event - as is the case in material fields which prohibit inertial motion, i.e. prohibit free fall - such time can't be realized as a coordinate. Nevertheless using such time as a scalar field enables to describe matter as acceleration fields and it allows new physics to emerge as a replacement of the stress-energy-momentum tensor. The punch line is electrogravity as a neat explanation of the Dark Matter effect and the advent of Sciama's Inertial Induction becomes realizable by separation of high electric charges. This paper totally rules out any measurable Biefeld Brown effect in vacuum on Pico-Farad or less, lonocrafts due to insufficient amount of electric charges. The electro-gravitational effect is due to field divergence and not directly due to intensity or gradient of the square norm. Inertial motion prohibition by material fields, e.g. intense electrostatic field, can be measured as mass dependent force on neutral particles that have rest mass and thus can measure proper time. Such acceleration should be measured in very low capacitance capacitors in order to avoid electro-gravitational effect. The acceleration should be from the positive to the negative charges. The electro-gravitational effect is opposite in direction, requires large amounts of separated charge carriers and acts on the entire negative to positive dipole. 


\section{ACKNOWLEDGEMENTS}

First, I should thank Dr. Sam Vaknin for his full support and for his ground breaking dissertation from 1982. Also thanks to professor Larry (Laurence) Horowitz from Tel Aviv university for his guidelines on an Early work. His letter from Wed, Jul 23, 2008, encouraged me to continue my research which started in 2003 and led to this paper. Special thanks to professor David Lovelock for his online help in understanding the calculus of variations and in fixing an error several years ago. Thanks to Elad Dayan, Ran Timar and Benny Versano for their help in understanding how profoundly difficult it is to separate charges as required by the idea of electro-gravity. Thanks to Mr. Yossi Avni who approved my research in 2003 into minimum cost diffeomorphism as part of a pattern matching algorithm. This early research helped in acquiring special knowledge in geometry that was later used in the presented theory.

Also a historical justice with the philosopher Rabbi Joseph Albo, Circa 1380-1444 must be made. In his book of principles, essay 18 appears to be the first known historical account of what Measurable Time - In Hebrew "Zman Moshoar" and Immeasurable Time "Zman Bilti Moshoar are. His Idea of the immeasurable time as a limit [18], is the very reason for an 11 years of research and for this paper.

\section{APPENDIX - The time field in the Schwarzschild solution}

Motivation: To make the reader familiar with the idea of maximal proper time and to calculate the background scalar time field of the Schwarzschild solution.

We would like to calculate $\left(\frac{\left(P^{\lambda} P_{\lambda}\right){ }_{m}\left(P^{s} P_{s}\right)_{{ }_{k}} g^{m k}}{\left(P^{i} P_{i}\right)^{2}}-\frac{\left(\left(P^{\lambda} P_{\lambda}\right){ }_{m} P^{m}\right)^{2}}{\left(P^{i} P_{i}\right)^{3}}\right)$ in Schwarzschild coordinates for a freely falling particle. This theory predicts that where there is no matter, the result must be zero. The result also must be zero along any geodesic curve but in the middle of a hollowed ball of mass the gradient of the absolute maximum proper time from "Big Bang" event or events, derivatives by space must be zero due to symmetry which means the curves come from different directions to the same event at the center. Close to the edges, gravitational lenses due to granularity of matter become crucial. The speed $U$ of a falling particle as measured by an observer in the gravitational field is 
$V^{2}=\frac{U^{2}}{C^{2}}=\frac{R}{r}=\frac{2 G M}{r C^{2}}$

Where $R$ is the Schwarzschild radius. If speed $V$ is normalized in relation to the speed of light then $V=\frac{U}{C}$. For a far observer, the deltas are denoted by $d t^{\prime}, d r^{\prime}$ and,

$$
\dot{r}^{2}=\left(\frac{d r}{d t}\right)^{2}=V^{2}\left(1-\frac{R}{r}\right)
$$

because $d r=d r^{\prime} / \sqrt{1-R / r}$ and $d t=d t^{\prime} \sqrt{1-R / r}$.

$$
\begin{aligned}
& P=\int_{0}^{t} \sqrt{\left(1-\frac{R}{r}\right)-\frac{(d r / d t)^{2}}{\left(1-\frac{R}{r}\right)}} d t=\int_{0}^{t} \sqrt{\left(1-\frac{R}{r}\right)-\frac{\frac{R}{r}\left(1-\frac{R}{r}\right)^{2}}{\left(1-\frac{R}{r}\right)}} d t=\int_{0}^{t} \sqrt{\left(1-\frac{R}{r}\right)^{2}} d t= \\
& \int_{0}^{t}\left(1-\frac{R}{r}\right) d t
\end{aligned}
$$

Which results in,

$$
P_{t}=\frac{d P}{d t}=\left(1-\frac{R}{r}\right)
$$

Please note, here $t$ is not a tensor index and it denotes derivative by $t ! ! !$

On the other hand

$$
\begin{aligned}
& P=\int_{\infty}^{r} \sqrt{\left(1-\frac{R}{r}\right) \frac{1}{\dot{r}^{2}}-\frac{1}{\left(1-\frac{R}{r}\right)}} d r=\int_{\infty}^{r} \sqrt{\frac{\left(1-\frac{R}{r}\right) \frac{r}{R}}{\left(1-\frac{R}{r}\right)^{2}}-\frac{1}{\left(1-\frac{R}{r}\right)}} d r=\int_{\infty}^{r} \sqrt{\frac{\frac{r-R}{R}}{\frac{r-R}{r}}} d r= \\
& \int_{\infty}^{r} \sqrt{\frac{r}{R}} d r
\end{aligned}
$$

Which results in

$$
P_{r}=\frac{d P}{d r}=\sqrt{\frac{r}{R}}
$$

Please note, here $r$ is not a tensor index and it denotes derivative by $r$ !!!

For the square norms of derivatives we use the inverse of the metric tensor,

So we have $\left(1-\frac{R}{r}\right) \rightarrow \frac{1}{\left(1-\frac{R}{r}\right)}$ and $\frac{1}{\left(1-\frac{R}{r}\right)} \rightarrow\left(1-\frac{R}{r}\right)$ 
So we can write

$$
\begin{aligned}
& N^{2}=P^{\lambda} P_{\lambda}=\left(1-\frac{R}{r}\right) P_{r}^{2}-\frac{1}{1-\frac{R}{r}} P_{t}^{2}=\left(1-\frac{R}{r}\right)\left(\frac{r}{R}-1\right)=\frac{r}{R}+\frac{R}{r}-2 \\
& N^{2}=\frac{r}{R}+\frac{R}{r}-2 \\
& N_{\lambda}^{2}=\frac{d N^{2}}{d x^{\lambda}} \text { And we can calculate } \\
& \frac{N_{\lambda}^{2} N^{2 \lambda}}{\left(N^{2}\right)^{2}}=\frac{\left(1-\frac{R}{r}\right)^{2}\left(\frac{1}{R}-\frac{R}{r^{2}}\right)^{2}}{\left(\frac{r}{R}+\frac{R}{r}-2\right)^{2}}
\end{aligned}
$$

We continue to calculate

$$
\begin{aligned}
& N^{2}{ }_{t} P_{t}=\left(1-\frac{R}{r}\right)^{2}\left(\frac{1}{R}-\frac{R}{r^{2}}\right) \sqrt{\frac{R}{r}} \text { and } \\
& \frac{N^{2} P_{t}}{\left(1-\frac{R}{r}\right)}=\left(1-\frac{R}{r}\right)\left(\frac{1}{R}-\frac{R}{r^{2}}\right) \sqrt{\frac{R}{r}}
\end{aligned}
$$

Please note, here $t$ is not a tensor index and it denotes derivative by $t$ !!!

$$
\left(1-\frac{R}{r}\right) N^{2}{ }_{r} P_{r}=\left(1-\frac{R}{r}\right)\left(\frac{1}{R}-\frac{R}{r^{2}}\right) \sqrt{\frac{r}{R}}
$$

Please note, here $r$ is not a tensor index and it denotes derivative by $r$ !!!

$$
\begin{aligned}
& N_{\lambda}^{2} P^{\lambda}=\left(1-\frac{R}{r}\right)\left(\frac{1}{R}-\frac{R}{r^{2}}\right)\left(\sqrt{\frac{r}{R}}-\sqrt{\frac{R}{r}}\right) \text { And } \\
& \left(N_{\lambda}^{2} P^{\lambda}\right)^{2}=\left(1-\frac{R}{r}\right)^{2}\left(\frac{1}{R}-\frac{R}{r^{2}}\right)^{2}\left(\frac{r}{R}+\frac{R}{r}-2\right)
\end{aligned}
$$

So

$$
\frac{\left(N_{\lambda}^{2} P^{\lambda}\right)^{2}}{\left(N^{2}\right)^{3}}=\frac{\left(1-\frac{R}{r}\right)^{2}\left(\frac{1}{R}-\frac{R}{r^{2}}\right)^{2}}{\left(\frac{r}{R}+\frac{R}{r}-2\right)^{2}}
$$

And finally, from (53) and (57) we have, 


$$
\begin{aligned}
& \left(\frac{\left(\mathrm{P}^{\lambda} \mathrm{P}_{\lambda}\right),_{\mathrm{m}}\left(\mathrm{P}^{s} \mathrm{P}_{s}\right)_{{ }_{\mathrm{k}}} g^{m k}}{\left(\mathrm{P}^{\mathrm{i}} \mathrm{P}_{\mathrm{i}}\right)^{2}}-\frac{\left(\left(\mathrm{P}^{\lambda} \mathrm{P}_{\lambda}\right),_{\mathrm{m}} \mathrm{P}^{m}\right)^{2}}{\left(\mathrm{P}^{\mathrm{i}} \mathrm{P}_{\mathrm{i}}\right)^{3}}\right)= \\
& \frac{N_{\lambda}^{2} N^{2^{\lambda}}}{\left(N^{2}\right)^{2}}-\frac{\left(N^{2}{ }_{\lambda} P^{\lambda}\right)^{2}}{\left(N^{2}\right)^{3}}= \\
& \frac{\left(1-\frac{R}{r}\right)^{2}\left(\frac{1}{R}-\frac{R}{r^{2}}\right)^{2}}{\left(\frac{r}{R}+\frac{R}{r}-2\right)^{2}}-\frac{\left(1-\frac{R}{r}\right)^{2}\left(\frac{1}{R}-\frac{R}{r^{2}}\right)^{2}}{\left(\frac{r}{R}+\frac{R}{r}-2\right)^{2}}=0
\end{aligned}
$$

which shows that indeed the gradient of time measured, by a falling particle until it hits an event in the gravitational field, has zero curvature as expected.

\section{APPENDIX - Acceleration Field Representation}

Motivation: To describe a field that interacts with all particles that have rest masses and not only with a particle that follows the gradient $\frac{\mathrm{P}_{\lambda}}{\sqrt{N^{2}}}$.

The acceleration can be expressed in coordinate dependent way by at least 3 variables $a, b, c$

$$
\begin{aligned}
& A_{\mu \nu}=\left(\begin{array}{cccc}
0 & a & -b & -c \\
-a & 0 & c & -b \\
b & -c & 0 & -a \\
c & b & a & 0
\end{array}\right) \text { such that } \\
& \left(\begin{array}{cccc}
0 & a & -b & -c \\
-a & 0 & c & -b \\
b & -c & 0 & -a \\
c & b & a & 0
\end{array}\right)\left(\begin{array}{l}
\frac{p^{0}}{\sqrt{p_{\mu} p^{\mu}}} \\
\frac{p^{1}}{\sqrt{p_{\mu} p^{\mu}}} \\
\frac{p^{2}}{\sqrt{p_{\mu} p^{\mu}}} \\
\frac{p^{3}}{\sqrt{p_{\mu} p^{\mu}}}
\end{array}\right)=\left(\begin{array}{l}
\frac{1}{2}\left(\frac{\left(P^{\lambda} P_{\lambda}\right)_{0}}{P^{i} P_{i}}-\frac{\left(P^{\lambda} P_{\lambda}\right)_{\mu_{\mu}} P^{\mu}}{\left(P^{i} P_{i}\right)^{2}} P_{0}\right) \\
\frac{1}{2}\left(\frac{\left(P^{\lambda} P_{\lambda}\right)_{1}}{P^{i} P_{i}}-\frac{\left(P^{\lambda} P_{\lambda}\right)_{\mu} P^{\mu}}{\left(P^{i} P_{i}\right)^{2}} P_{1}\right) \\
\frac{1}{2}\left(\frac{\left(P^{\lambda} P_{\lambda}\right)_{2}}{P^{i} P_{i}}-\frac{\left(P^{\lambda} P_{\lambda}\right)_{\mu} P^{\mu}}{\left(P^{i} P_{i}\right)^{2}} P_{2}\right) \\
\frac{1}{2}\left(\frac{\left(P^{\lambda} P_{\lambda}\right)_{3}}{P^{i} P_{i}}-\frac{\left(P^{\lambda} P_{\lambda}\right)_{\mu} P^{\mu}}{\left(P^{i} P_{i}\right)^{2}} P_{3}\right)
\end{array}\right)
\end{aligned}
$$

$$
\left(\begin{array}{cccc}
0 & a & -b & -c \\
-a & 0 & c & -b \\
b & -c & 0 & -a \\
c & b & a & 0
\end{array}\right)\left(\begin{array}{c}
r^{0} \\
r^{1} \\
r^{2} \\
r^{3}
\end{array}\right)=a\left(\begin{array}{c}
r_{1} \\
-r_{0} \\
-r_{3} \\
r_{2}
\end{array}\right)+b\left(\begin{array}{c}
-r_{2} \\
-r_{3} \\
r_{0} \\
r_{1}
\end{array}\right)+c\left(\begin{array}{c}
-r_{3} \\
r_{2} \\
-r_{1} \\
r_{0}
\end{array}\right)
$$


As the reader can see, the vectors are not perpendicular in Minkowsky geometry but they are perpendicular in ordinary Euclidean geometry. These vectors are closely related to Ashtekar variables [19].

Let $A$ denote $A_{\mu \nu}$. Obviously $A A^{*}=\left(a a^{*}+b b^{*}+c c^{*}\right) I$ where $I$ is the identity matrix and if $a, b, c$ are real numbers and $g_{\mu \nu} \approx \delta_{\mu \nu}=I_{\mu \nu}$ then the determinant is

$\operatorname{Det}(\mathrm{A})=\left(a^{2}+b^{2}+c^{2}\right)^{2} \approx\left(\frac{1}{2}\left(U_{i} U *^{i}+U *_{i} U^{i}\right)\right)^{2}$.

Such that $U_{i}$ is the complex form of the curvature vector where the scalar field $p$ is a multiplication of the time field of upper limit of measurable time from near the big bang singularity event or manifold of events from which we can say the cosmos started to expand $p=\tau \psi . \psi$ is the wave function describing the material observer of the time field.

We can write a local coordinate representation of $A_{\mu \nu}$ as a linear combination of Quaternions,

$A_{\mu v}=a A 1_{\mu v}+b A 2_{\mu v}+c A 3_{\mu \nu}$

$A 1=\left(\begin{array}{cccc}0 & 1 & 0 & 0 \\ -1 & 0 & 0 & 0 \\ 0 & 0 & 0 & -1 \\ 0 & 0 & 1 & 0\end{array}\right), A 2=\left(\begin{array}{cccc}0 & 0 & -1 & 0 \\ 0 & 0 & 0 & -1 \\ 1 & 0 & 0 & 0 \\ 0 & 1 & 0 & 0\end{array}\right), A 3=\left(\begin{array}{cccc}0 & 0 & 0 & -1 \\ 0 & 0 & 1 & 0 \\ 0 & -1 & 0 & 0 \\ 1 & 0 & 0 & 0\end{array}\right)$

$I=\left(\begin{array}{llll}1 & 0 & 0 & 0 \\ 0 & 1 & 0 & 0 \\ 0 & 0 & 1 & 0 \\ 0 & 0 & 0 & 1\end{array}\right)$

$A 1 \cdot A 2=A 3, A 1 \cdot A 3=-A 2, A 2 \cdot A 3=A 1, A 2 \cdot A 1=-A 3, A 3 \cdot A 1=A 2, A 3 \cdot A 2=-A 1$ , $A 1 \cdot A 1=-I, A 2 \cdot A 2=-I, A 3 \cdot A 3=-I$.

\section{APPENDIX - Event Theory}

Motivation: To provide an alternative integration constraint that leads to a new possible theory.

An interesting alternative to (24.1) is that $\psi$ is not a particle wave function but an event function, i.e. a collision with a particle in a 4 dimensional space-time, 
$\int_{\Omega^{4}(\tau)} \psi \psi * \sqrt{-g} d \Omega^{4}=1$

And instead of $P=\tau \psi$ we choose $P=\sqrt{\tau} \psi$. The reader can verify that the same curvature vector in (23) is reached by the assignment $P=\sqrt{\tau} \psi$ if $\psi$ depends only on $\tau$. Then from (24.2) we get a new random variable $\tau_{\text {Event }}$

$\tau_{\text {Event }}=\int_{\Omega^{4}(\tau)} \tau \psi \psi * \sqrt{-g} d \Omega^{4}=\int_{\Omega^{4}(\tau)} P P * \sqrt{-g} d \Omega^{4}$

and adding this constraint to the complex form of (24) we have the following variations system,

$$
\begin{aligned}
& P=\sqrt{\tau} \psi \\
& \mathrm{Z}=N^{2}=\frac{\mathrm{P}_{\mu} \mathrm{P}^{* \mu}+\mathrm{P}_{\mu}^{*} \mathrm{P}^{\mu}}{2} \text { and } U_{\lambda}=\frac{Z_{\lambda}}{Z}-\frac{Z_{k} P^{* k} P_{\lambda}}{Z^{2}} \text { and } \\
& \mathrm{L}=\frac{1}{4}\left(\frac{1}{2}\left(\mathrm{U}^{\mathrm{k}} \mathrm{U}^{*}{ }_{\mathrm{k}}+\mathrm{U}^{* \mathrm{k}} \mathrm{U}_{\mathrm{k}}\right)\right) \\
& R=\text { Riccicurvature, } \\
& \text { Min Action }=\operatorname{Min} \int_{\Omega}\left(\frac{1}{2} R-8 \pi L+\lambda P P^{*}\right) \cdot \sqrt{-g} d \Omega \\
& \text { and } \\
& \int_{\Omega} P P^{*} \cdot \sqrt{-g} d \Omega=\tau_{\text {Event }} \\
& \lambda=\text { Const. }
\end{aligned}
$$

(64) is worthy of further research. To prove that (64) is consistent with quantum mechanics, a family of solutions to (64) with complex functions $\psi_{\tau}$ should exist such that $\tau_{\text {Event }}$ will be increasing and will describe detection events of the same particle.

\section{REFERENCES}

[1] S. Vaknin, "Time Asymmetry Revisited"[microform], 1982, Library of Congress Doctorate Dissertation, Microfilm 85/871 (Q).

[2] E. H. Suchard, "Upper Time Limit, Its Gradient Curvature and Matter", Journal of Modern Physics and Applications, received Sep., 24, 2003, 2014, 2014:5, ISSN 2051-5480 
[3] John W. Milnor, Topology from the Differentiable Viewpoint, pages 32-41, ISBN 0-69104833-9

[4] Y. Friedman and T. Scarr, "Making the relativistic dynamics equation covariant: explicit solutions for motion under a constant force", Volume 86 Number 6, 2012 Physica Scripta 065008 doi:10.1088/0031-8949/86/06/065008

[5] T. Datta, M. Yin, A. Dimofte, M. C. Bleiweiss and Z. Cai, "Experimental Indications of Electro-Gravity", 8 Sep 2005 arXiv:physics/0509068[physics.gen-ph]

[6] Hector Luis Serrano, (President of Gravitec Inc.) capacitor at $1.72 \times 10^{\wedge}-6$ Torr, Experiment by NASA and Gravitec, Video from July 3, 2003.

[7] Elie Cartan, The Theory of Spinors, Dover Publications Inc. Mineola, N.Y., ISBN 0-48664070-1, p. $149(14)$

[8] E. E. Escultura, Chaos, Turbulence and Fractal: Theory and Applications, International Journal of Modern Nonlinear Theory (SCIRP), pp. 176 - 185; http://www.scirp.org/journal/PaperInformation.aspx?PaperlD=36849; doi: 10.4236/ijmnta.2013.23025.

[9] Victor Guillemin / Alan Pollack, Differential Topology, Prentice-Hall, Englewood Cliffs, N.J.,1974. pp. 39-48, Sard's Theorem and Morse Functions, ISBN 0-13-212605-2

[10] Victor Guillemin / Alan Pollack, Differential Topology, Submersions, Page 20, ISBN 0-13212605-2

[11] Victor Guillemin / Alan Pollack, Differential Topology, Submersions, Page 21, Preimage Theorem, ISBN 0-13-212605-2

[12] D. Lovelock and H. Rund, Tensors, Differential Forms and Variational Principles, Dover 
Publications Inc. Mineola, N.Y., ISBN 0-486-65840-6, p. 262, 3.27

[13] D. Lovelock and H. Rund, Tensors, Differential Forms and Variational Principles, Dover Publications Inc. Mineola, N.Y., ISBN 0-486-65840-6, p. 261, 3.26

[14] D. Lovelock and H. Rund, "The Numerical Relative Tensors", Tensors, Differential Forms and Variational Principles, 4.2, Dover Publications Inc. Mineola, N.Y. , ISBN 0-486-65840-6, p. $113,2.18$, p. $114,2.30$

[15] D.W. Sciama, "On the Origin of Inertia", Monthly Notices of the Royal Astronomical Society, Vol. 113, p.34, August 1952

[16] M. Alcubierre, "The warp drive: hyper-fast travel within general relativity", Class.Quant.Grav.11:L73-L77, 1994, DOI: 10.1088/0264-9381/11/5/001

[17] Thomas B. Bahder and Chris Fazi, "Force on an Asymmetric Capacitor", Army Research Laboratory ARL, March 2003, site: http://arxiv.org/ftp/physics/papers/0211/0211001.pdf [18] J. Albo, Book of Principles (Sefer Ha-ikarim), "Immeasurable time - Maamar 18", "measurable time by movement". (Circa 1380-1444, unknown), The Jewish publication Society of America (1946), ASIN: B001EBBSIC, Chapter 2, Chapter 13.

[19] A. Ashtekar, New Variables for Classical and Quantum Gravity, Physical Review Letters, 57, (1986), pp. 2244-2247 